\title{
Evolution of Glaucoma Research: A Scientometric Review
}

\author{
Parul Ichhpujani ${ }^{1}$, Gagan Kalra ${ }^{2}$, Rishemjit Kaur ${ }^{3}$, Shibal Bhartiya ${ }^{4}$
}

\begin{abstract}
Ophthalmic literature has been subjected to scientometrics in the past both for specific disease pathologies, such as, age-related macular degeneration, glaucoma, and diabetic retinopathy, and specific journals to add insight to the evolving trends. This short scientometric review looks at the distribution pattern and subject domain knowledge of worldwide glaucoma research with data extracted from Web of Science (WoS, Clarivate Analytics) for the past 74 years.

Keywords: Bibliometrics, Citation, Collaboration, Glaucoma, Scientometric analysis.

Journal of Current Glaucoma Practice (2020): 10.5005/jp-journals-10078-1286
\end{abstract}

\section{INTRODUCTION}

Over the years, the concept of glaucoma has evolved from being primarily attributed to elevated intraocular pressure (IOP) to the development of optic neuropathy as the central concept of glaucoma. Despite a vast amount of research, the definition and pathogenesis of glaucoma remains controversial and is still being explored. Analysis of research evolution and research evaluation methodology is as important as the research topics and can be studied using the scientific literature produced in the research field.

Over the years, researchers have coined various terminologies for information and literature analysis, such as, bibliometrics, informetrics, scientometrics, and webometrics.

Bibliometric analysis is based on the identification of the corpus of literature, i.e., exploring multiple aspects of publications (e.g., number of papers, growth of literature), within a given subject area. It studies the relationship between numbers and patterns in bibliographic data, library, and database usage. Scientometrics is a subfield of bibliometrics, which involves the assessment of the impact of research papers and academic journals, the understanding of scientific citations, and the use of such measurements in furthering research in the right direction. Informetrics is a broad term that encompasses modeling, reasoning, and drawing inferences under conditions of noisy and limited information. Webometrics examines different aspects of the web, such as, link analysis, web citation, search engines, etc.

The major scientometric analysis pertaining to glaucoma that we came across had data extracted from the Web of Science (WoS), Institute for Scientific Information (ISI), and mapped 20 years of glaucoma research.' In this short scientometric review, we looked at the distribution pattern and subject domain knowledge of worldwide glaucoma research with data extracted from the WoS (Clarivate Analytics) for the past 74 years.

\section{Materials and Methods}

The current descriptive scientometric study targeted a schematic view of the scientific map in the field of glaucoma. We used a WoS (https://webofknowledge.com), as our database for our analysis on January 1, 2020, using the institutional access (by RK). The term used for the search was chosen in accordance with Medical Subject Heading (Mesh) which is used to index PubMed@ contents. Only one parent term (glaucom*) was used. We searched for glaucom*

\begin{abstract}
1,2Department of Ophthalmology, Government Medical College and Hospital, Chandigarh, India

${ }^{3}$ CSIR-Central Scientific Instruments Organisation, Chandigarh, India

${ }^{4}$ Glaucoma Facility, Department of Ophthalmology, Fortis Memorial Research Institute, Gurugram, Haryana, India

Corresponding Author: Parul Ichhpujani, Department of Ophthalmology, Government Medical College and Hospital, Chandigarh, India, Phone: +91 9501071591, e-mail: parul77@ rediffmail.com

How to cite this article: Ichhpujani P, Kalra G, Kaur R, et al. Evolution of Glaucoma Research: A Scientometric Review. J Curr Glaucoma Pract 2020;14(3):98-105.
\end{abstract}

Source of support: Nil

Conflict of interest: None

in the title of articles which ensured that the articles relevant to glaucoma research were included in the analysis. We did not search the abstract or introduction or any other section of the article. The "**" is a wildcard that can take any value. The query was done using all the variations of this term for the intended period of January 1945 to January 1, 2020.

The data were exported in BibTeX (.bib) file format. This BibTeX file was further used as the data source in the Bibliometrix $\mathrm{R}$ library and biblioshiny app. The retrieved documents included reviews, proceeding papers, letters, editorial material, meeting abstracts, notes, reprints, corrections, news items, book reviews, and additions. Articles about glaucoma were analyzed regarding the topics' structure, history, and document relationships. Also, the trends in the most influential publications and authors were analyzed.

\section{Results}

Our WoS query returned a total of 32,551 documents that were published between 1945 and 2019 and were obtained from 1,906 sources. The total annual production trends along with annual average article citation trends have been summarized in Figure 1.

\section{Geographic Metrics}

United States of America (USA) $(n=7,605,23 \%)$, China $(n=1,472$, $5 \%)$, United Kingdom (UK) $(n=1,466,4 \%)$, India $(n=1,375,4 \%)$, 


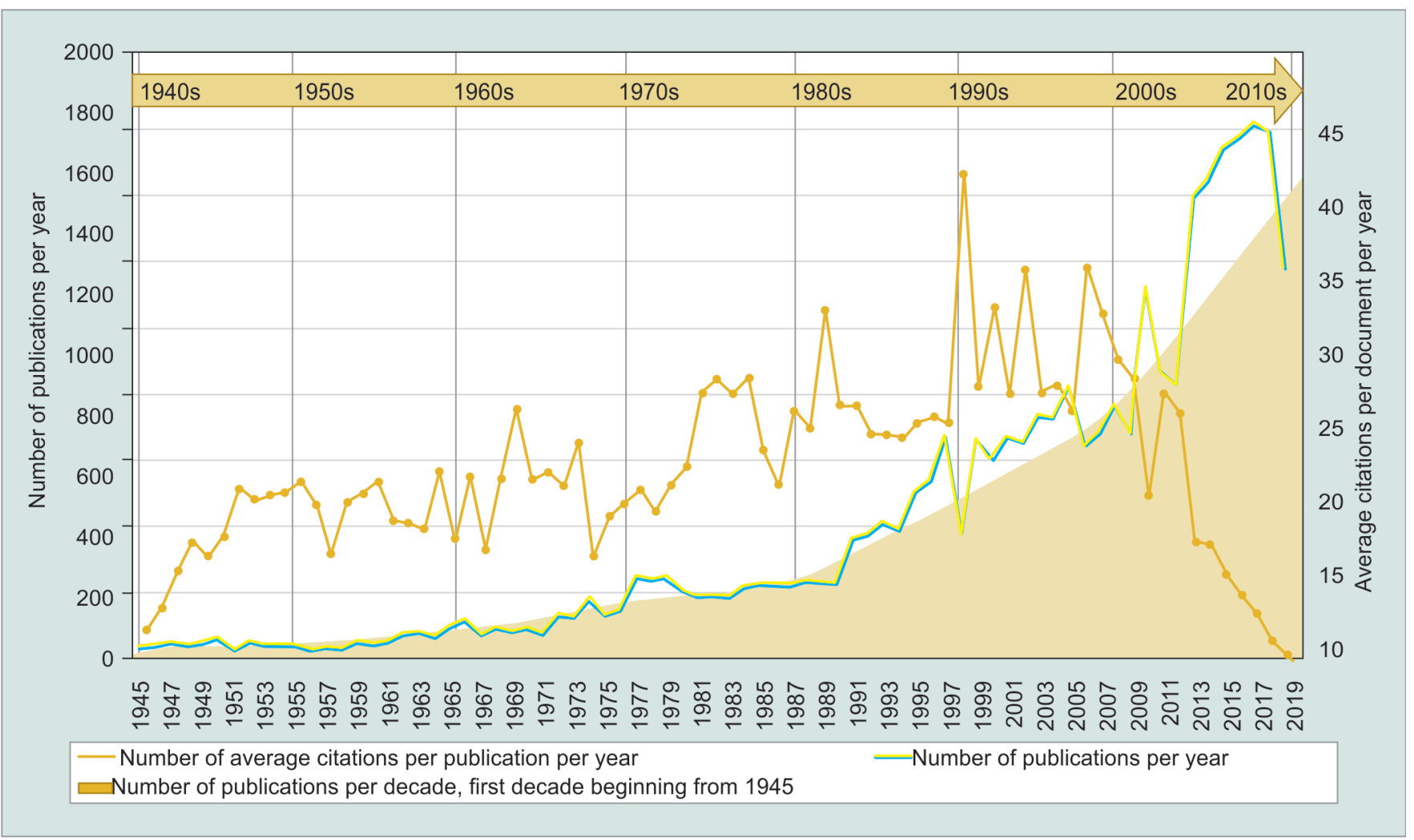

Fig. 1: Annual production trends along with annual average article citation trends

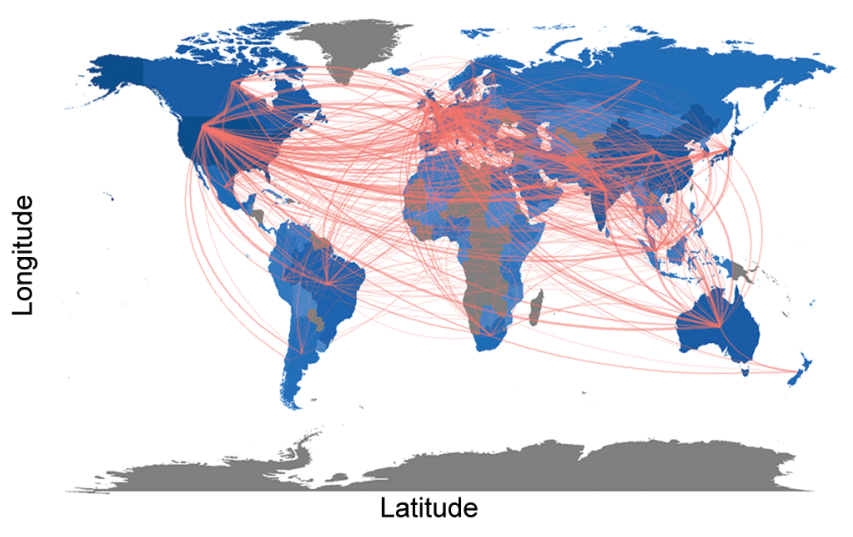

Fig. 2: Global collaboration network over the period of 1945 to 2019

and Germany ( $n=1,271,4 \%)$ were the highest producing countries among others. Global collaboration network over the period analyzed in the study is represented in Figure 2. The USA has maximum collaborations with China, Brazil, Canada, and Japan whereas India collaborates mostly with Australia, UK, Singapore, etc. Table 1 shows the top 20 country-wise article productivity and collaboration. International collaboration was defined as an article being published by at least two authors with two different country affiliations.

\section{Source Metrics}

Hirsch index (h-index) and a total number of citations (TC; average citation count per article) are two important author-level parameters. $\mathrm{h}$-index is a metric that attempts to measure both the productivity and citation impact of the publications of a scientist.
Table 1: Top 20 country-wise article productivity and collaboration

\begin{tabular}{lcrrl}
\hline Country & Articles & \multicolumn{1}{l}{ SCP } & MCP & MCP ratio \\
\hline USA & 7,605 & 6,438 & 1,167 & 0.1535 \\
China & 1,472 & 1,221 & 251 & 0.1705 \\
United Kingdom & 1,466 & 1,195 & 271 & 0.1849 \\
India & 1,375 & 1,167 & 208 & 0.1513 \\
Germany & 1,271 & 1,088 & 183 & 0.1440 \\
Japan & 1,107 & 1,046 & 61 & 0.0551 \\
Korea & 867 & 782 & 85 & 0.0980 \\
Italy & 657 & 569 & 88 & 0.1339 \\
Canada & 552 & 429 & 123 & 0.2228 \\
Australia & 548 & 405 & 143 & 0.2609 \\
France & 515 & 465 & 50 & 0.0971 \\
Turkey & 409 & 399 & 10 & 0.0244 \\
Spain & 352 & 300 & 52 & 0.1477 \\
Switzerland & 305 & 233 & 72 & 0.2361 \\
Singapore & 265 & 138 & 127 & 0.4792 \\
Brazil & 237 & 204 & 33 & 0.1392 \\
Netherlands & 230 & 186 & 44 & 0.1913 \\
Sweden & 207 & 176 & 31 & 0.1498 \\
Israel & 199 & 166 & 33 & 0.1658 \\
Greece & 184 & 123 & 61 & 0.3315 \\
\hline SCP, single coun & & 132 &
\end{tabular}

SCP, single country publication (intracountry); MCP, multiple country publication (intercountry); MCP ratio, refers to MCP as a proportion of total publication number

Ophthalmology (h-index $=125, \mathrm{TC}=70,299$ ), Investigative Ophthalmology and Visual Science (IOVS) (h-index =107, TC=49,537), 
Archives of Ophthalmology (Now, JAMA Ophthalmology) (h-index $=99, \mathrm{TC}=48,594$ ), American Journal of Ophthalmology (AJO) (h-index $=98, T C=50,184$ ), and British Journal of Ophthalmology (BJO) ( $h$-index $=77, \mathrm{TC}=35,345$ ) were the highest impact sources for glaucoma research among others.

In terms of total article production, IOVS ( $n=7,011,21 \%)$, Journal of Glaucoma ( $n=1,807,6 \%)$, AJO ( $n=1,747,5 \%)$, Ophthalmology $(n=1,412,4 \%)$, and BJO $(n=1,134,3 \%)$ had the greatest number of research items pertaining to our topic of interest.

The top 20 journals in terms of impact along with their number of research items pertaining to glaucoma have been summarized in Table 2.

\section{Bradford's Law of Scattering}

Bradford's law of scattering helps to identify the core journals relevant to the research field (Fig. 3). It is based on the principle of centric productivity zones, where the journals are divided into different zones having the same number of articles. The core journals are those that contribute to one-third of total publications. ${ }^{2}$ Top four core journals identified were IOVS, Journal of Glaucoma, AJO, and Ophthalmology.

\section{Author Metrics}

Harry Quigley (h-index $=78, \mathrm{TC}=28,237, n=278,0.85 \%$ ), Robert Weinreb (h-index $=68, \mathrm{TC}=16,495, n=523,1.6 \%$ ), Robert Ritch (h-index $=54, \mathrm{TC}=12,607, n=386,1.2 \%$ ), Stephen Drance (h-index $=53, \mathrm{TC}=9,114, n=175,0.53 \%$ ), and Linda M Zangwill (h-index = $51, \mathrm{TC}=6,933, n=243,0.74 \%$ ) were the leading authors by impact among others in the area of study.

The top 10 cited documents, authors, local and global citation count metrics along with other parameters are summarized in Table 3. Table 4 elucidates the author impact of 20 top authors.

\section{Lotka's Law}

This most basic law of bibliometrics deals with the frequency of publications by authors in any given field. It helps to examine the productivity of authors. It states that the relationship between the number of authors and publications follows an inverse square law, which means that most of the authors publish a few papers, while the authors publishing many papers are few. Lotka's law is given by the following equation:

$$
X^{\beta} Y=C
$$

where $Y$ is the proportion of authors producing $X$ publications, and

$\beta$ coefficient and $C$ are constants specific to the research field. Lotka found that almost $60 \%$ of authors make a single contribution and $15 \%$ will have two contributions. ${ }^{3}$

Table 5 shows the observed distribution of publications by authors in the field of glaucoma. It can be observed that $58.3 \%$ of authors have published only 1 article, 17.7\% have published 2 articles while only $3.6 \%$ of authors have produced more than 10 articles. We have estimated the $\beta$ coefficient of our bibliometric collection using Lotka's law. The estimated $\beta$ coefficient was 1.93 (with the goodness of fit $=0.92$ ) and the constant $C$ was 0.361 . The $p$ value of 0.008 was obtained by employing the KolmogorovSmirnoff two-sample test indicating that there is no significant difference obtained between theoretical Lotka's and the observed distribution (Fig. 4).

\section{Document Metrics}

Global Citation Score (GCS) provides the citation frequency based on the full WoS count at the time the data were downloaded.

Table 2: Journal-wise productivity of top 20 journals

\begin{tabular}{|c|c|c|c|c|c|c|}
\hline S. no. & Journal name & $h$-Index & g-Index & m-Index & Total citations & Total publications \\
\hline 1 & Ophthalmology & 125 & 193 & 2.90697674418605 & 70,299 & 1,412 \\
\hline 2 & Investigative Ophthalmology and Visual Science & 107 & 151 & 2.43181818181818 & 49,537 & 7,011 \\
\hline 3 & Archives of Ophthalmology & 99 & 183 & 1.30263157894737 & 48,594 & 1,068 \\
\hline 4 & American Journal of Ophthalmology & 98 & 155 & 1.28947368421053 & 50,184 & 1,747 \\
\hline 5 & British Journal of Ophthalmology & 77 & 140 & 1.01315789473684 & 35,345 & 1,134 \\
\hline 6 & Journal of Glaucoma & 65 & 95 & 2.6 & 26,173 & 1,807 \\
\hline 7 & Survey of Ophthalmology & 55 & 87 & 1.25 & 8,689 & 200 \\
\hline 8 & Experimental Eye Research & 46 & 69 & 0.92 & 6,350 & 187 \\
\hline 9 & Acta Ophthalmologica & 43 & 58 & & 8,574 & 963 \\
\hline 10 & $\begin{array}{l}\text { Graefes Archive for Clinical and Experimental } \\
\text { Ophthalmology }\end{array}$ & 43 & 63 & & 8,396 & 492 \\
\hline 11 & Eye & 41 & 59 & 1.24242424242424 & 8,552 & 602 \\
\hline 12 & Molecular Vision & 38 & 50 & 1.9 & 5,977 & 280 \\
\hline 13 & Acta Ophthalmologica Scandinavica & 37 & 53 & 1.42307692307692 & 4,439 & 287 \\
\hline 14 & Journal of Cataract and Refractive Surgery & 36 & 60 & 1.02857142857143 & 4,504 & 214 \\
\hline 15 & Current Opinion in Ophthalmology & 36 & 57 & 1.16129032258065 & 4,077 & 189 \\
\hline 16 & PLoS One & 34 & 45 & 2.61538461538462 & 4,693 & 391 \\
\hline 17 & Ophthalmic Surgery and Lasers & 34 & 55 & 0.708333333333333 & 5,054 & 279 \\
\hline 18 & Clinical and Experimental Ophthalmology & 32 & 45 & & 4,253 & 553 \\
\hline 19 & Progress in Retinal and Eye Research & 32 & 47 & 1.18518518518519 & 5,435 & 47 \\
\hline 20 & Human Molecular Genetics & 30 & 50 & 1.2 & 3,862 & 50 \\
\hline
\end{tabular}




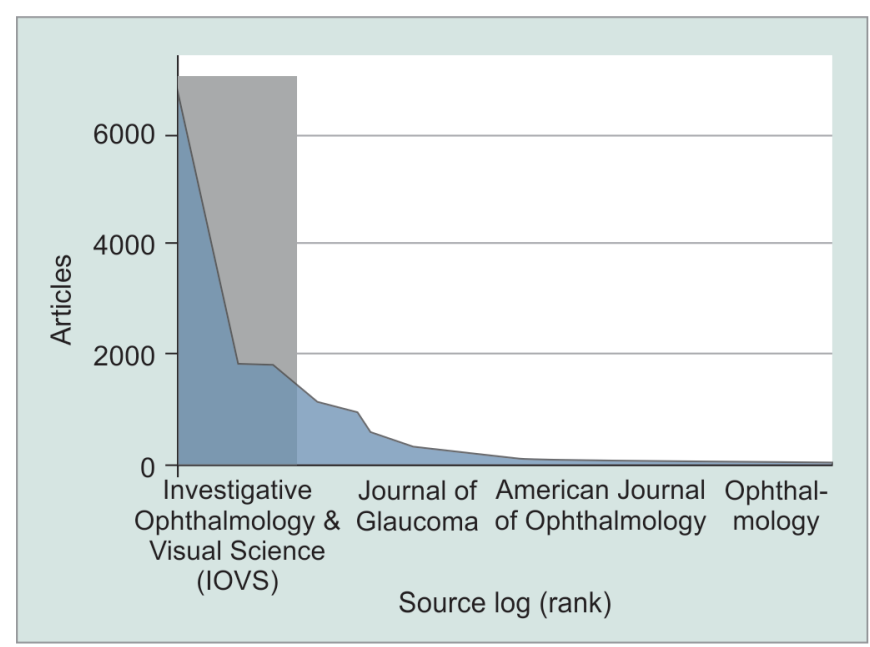

Fig. 3: Bradford's law

Harry Quigley, 2006 (GCS = 3,379, Country = USA), Michael A Kass, 2002 (GCS = 2,079, Country = USA), Anders Heijl 2002 (GCS $=1,727$, Country = Sweden), AGIS 2000 (GCS = 1,625, Country = USA), and Harry Quigley, 1996 (GCS = 1,499, Country = USA) had the greatest number of citations among others from our topic of interest as elucidated in Table 2.

\section{KeyWords Plus and Author Keywords}

KeyWords Plus ${ }^{\circledast}$ is unique to WoS and consists of words and phrases harvested from the titles of the cited articles. KeyWords Plus are searched in a Topic search. All author addresses are indexed and searchable. Open-angle glaucoma $(n=4,102)$, IOP $(n=3,015)$, ocular hypertension $(n=1,156)$, trabeculectomy $(n=1,141)$, and risk factors ( $n=913$ ) were the most commonly occurring keywords according to KeyWords Plus among others between 1990 and 2019. KeyWords Plus trends for the top 11 keywords are summarized in Figure 5.

Glaucoma $(n=5,459)$ was the most common keyword listed by authors. Besides "glaucoma", IOP $(n=1,159)$, trabeculectomy $(n=$ 488), primary open-angle glaucoma ( $n=377)$, ocular hypertension ( $n=329)$, and visual fields $(n=316)$ were the most commonly listed keywords by authors between 1990 and 2019. Author keywords trends for the top 11 keywords are summarized in Figure 6.

\section{Universities/Institutes with Significant Contribution}

Table 5 enlists the top 20 universities or institutes with a maximum contribution in terms of peer-reviewed, published glaucoma articles.

\section{Discussion}

We classified the subject matter of the top-cited articles into broad categories, such as, clinical, epidemiological, basic science, etc. Clinical (6/10) followed by epidemiological (3/10) and basic science research (1/10) formed the top 10 cited articles in our study. This is slightly different from the composition noted by Ramin et al. but generally follows a similar trend. ${ }^{1}$

Most of these top-cited articles in our study came from the USA (7/10). Most cited clinical articles generally focused on the control of IOP, risk factor identification, factors affecting treatment, and progression of the disease. This is also reflected in the preferential publication of analytical research involving case-control, cohort, or randomized trials in all of ophthalmology. ${ }^{4}$ Epidemiological

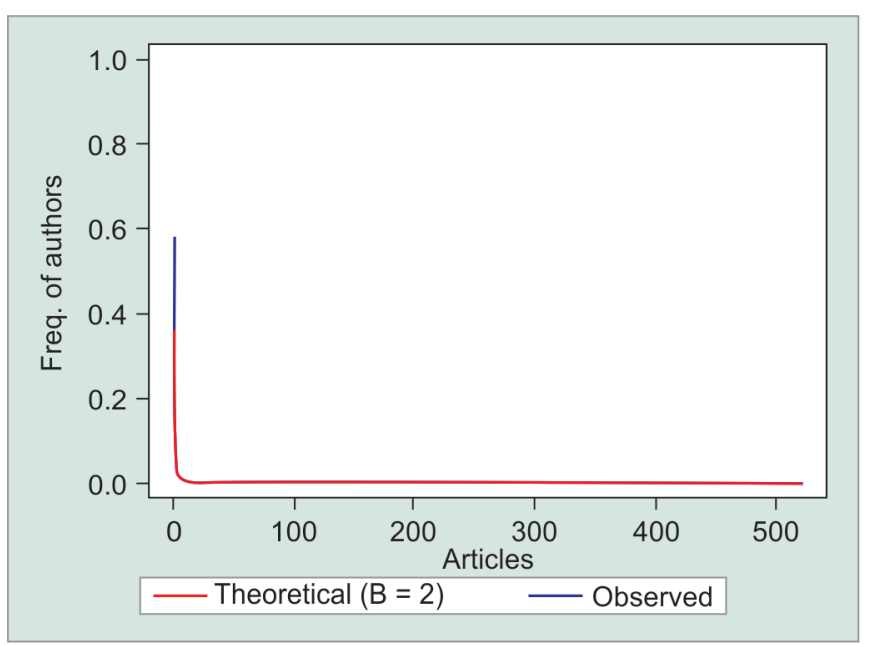

Fig. 4: Lotka's law

research pertaining to glaucoma prevalence were some of the highest cited articles in glaucoma research. Notably, Quigley et al. had two publications in the top-cited articles in glaucoma at the second- and third-highest number of citations as noted by Ramin et al. ${ }^{1}$ In our study, these publications have the top and fifth-highest citations, respectively. A relatively recent publication from Tham et al. projected glaucoma prevalence in the world till 2040 and is one of the highest cited glaucoma articles in the short 6 years of its publication. ${ }^{5}$

Here, we would like to highlight that generally, researchers work on a particular research area for some time and their work is extended in multiple articles. While self-citations are used to build over their previous work, they can sometimes be abused for self-promotional activities and increasing the citation impact. However, studies have shown that the self-citations do not impact the macro bibliometrics measure and hence, their exclusion was also deemed unnecessary. ${ }^{6,7}$ Also, self-citations have a diminishing impact with time.

More recently, keyword trends in both KeyWords Plus and Author keywords indicate an increasing interest in translational glaucoma research as reflected by an increase in the use of keywords "Optical Coherence Tomography", "Perimetry", "Visual Field Testing", and "Nerve fiber layer". This is in line with contemporary research albeit over shorter periods. ' With the advent of advanced imaging and testing techniques, new parallels are being drawn between translational research and clinical ophthalmology resulting in such trends.

It is a common notion that the most cited articles were perhaps published in the most impactful journals. But as our analysis shows, from the top 10 cited articles only one that is the seventh-highest cited article was published in Ophthalmology that was shown to be the highest impact journal in our analysis. Most other articles from our top 10 were published in third-, fourth-, and fifth-highest impact journals. This could be explained by the targeted nature of research conducted in the field of glaucoma within an already specialized branch of ophthalmology where most active researchers look to publish in highly specialized journals, so their work reaches their contemporaries in the field. ${ }^{8}$ Another study published in 2007 showed that the top 100 cited articles at the time were published in Archives of Ophthalmology (now JAMA Ophthalmology), followed by other higher impact journals. ${ }^{9}$ 


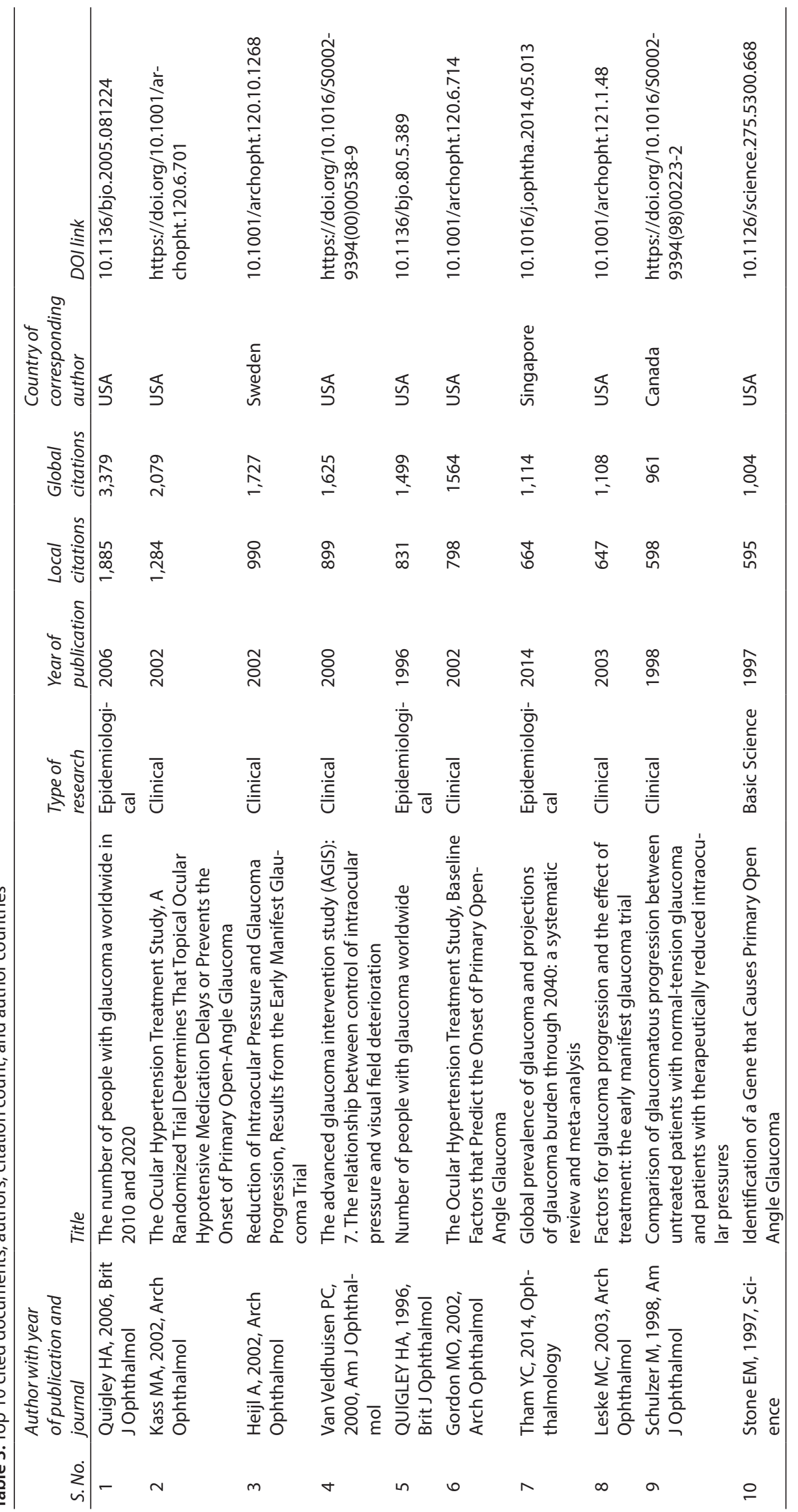


Evolution of Glaucoma Research: A Scientometric Review

Table 4: Author impact of top 20 authors

\begin{tabular}{|c|c|c|c|c|c|}
\hline Author & h-Index & g-Index & m-Index & $T C$ & $N P$ \\
\hline Quigley HA & 78 & 167 & 1.696 & 28,237 & 278 \\
\hline Weinreb RN & 68 & 110 & 1.619 & 16,495 & 523 \\
\hline Ritch R & 54 & 105 & 1.256 & 12,607 & 386 \\
\hline Drance SM & 53 & 93 & 0.869 & 9,114 & 175 \\
\hline Zangwill LM & 51 & 78 & 1.962 & 6,933 & 243 \\
\hline Caprioli J & 48 & 81 & & 7,405 & 265 \\
\hline Medeiros FA & 47 & 78 & 2.35 & 6,943 & 247 \\
\hline Sample PA & 46 & 68 & 1.314 & 4,843 & 125 \\
\hline Liebmann JM & 43 & 67 & 1.344 & 5,535 & 255 \\
\hline Hitchings RA & 43 & 69 & 0.956 & 5,302 & 191 \\
\hline Jonas JB & 42 & 65 & 1.273 & 5,164 & 174 \\
\hline Spaeth GL & 41 & 65 & 0.759 & 5,035 & 219 \\
\hline Friedman DS & 41 & 75 & 1.577 & 5,788 & 145 \\
\hline Flammer J & 38 & 69 & 0.927 & 5,092 & 121 \\
\hline Heijl A & 38 & 88 & 0.826 & 7,904 & 110 \\
\hline Aung $T$ & 36 & 71 & 1.565 & 5,740 & 248 \\
\hline Allingham RR & 35 & 57 & 1.061 & 3,655 & 159 \\
\hline Bengtsson B & 35 & 84 & 0.875 & 7,198 & 87 \\
\hline Wiggs JL & 34 & 56 & 1.172 & 3,460 & 179 \\
\hline Schuman JS & 34 & 59 & 1.172 & 3,683 & 151 \\
\hline
\end{tabular}

h-Index: An author has an $h$-index of " $h$ " when they have $h$ papers that have been cited $h$ times at least

$\mathrm{g}$-Index: Where the top " $\mathrm{g}$ " articles have together received " $\mathrm{g}$ " citations

$\mathrm{m}$-Index: $\mathrm{h}$-index divided by the number of years that a scientist has been active

NP, number of publications; TC: total citations

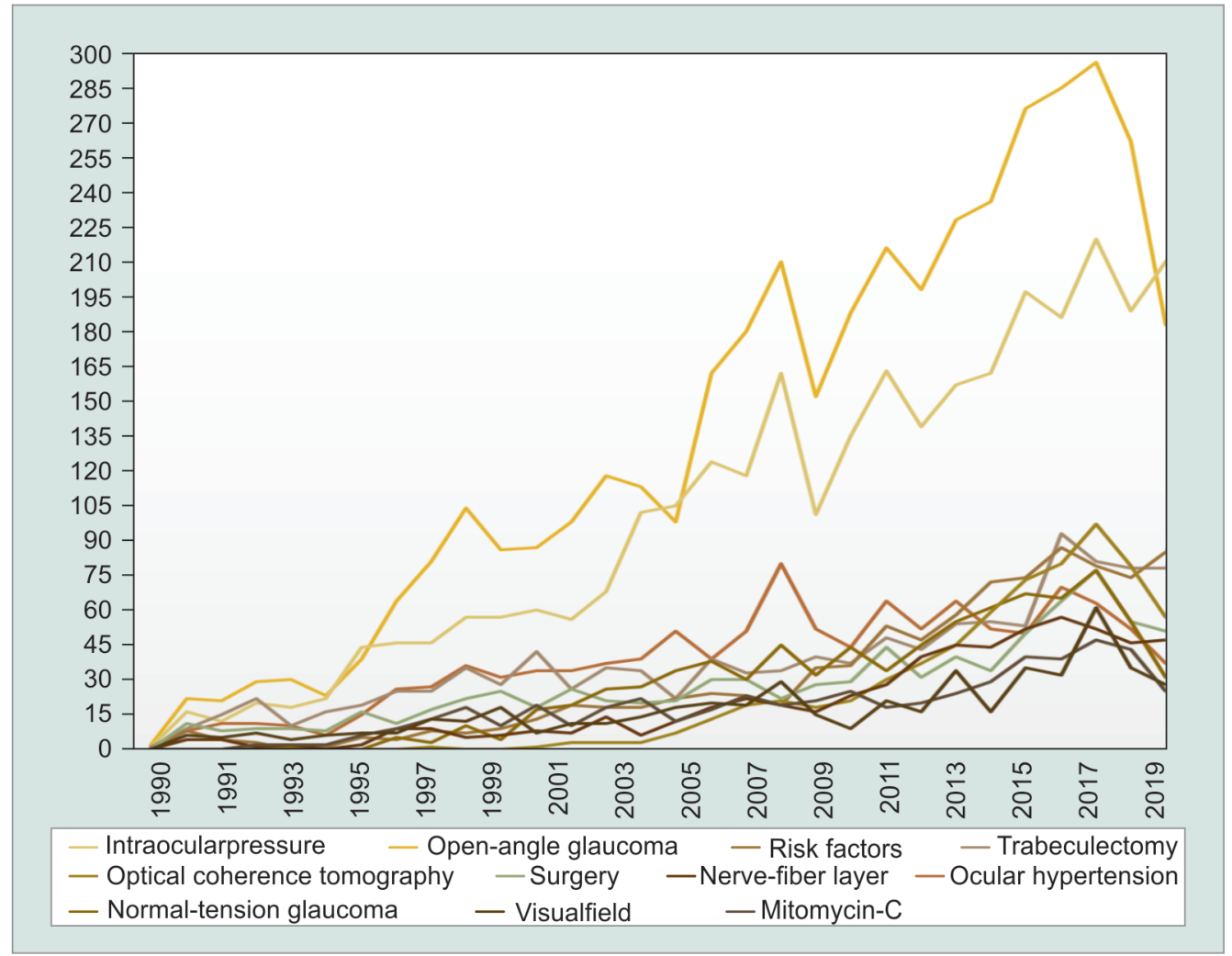

Fig. 5: KeyWords Plus trends for top 11 keywords 


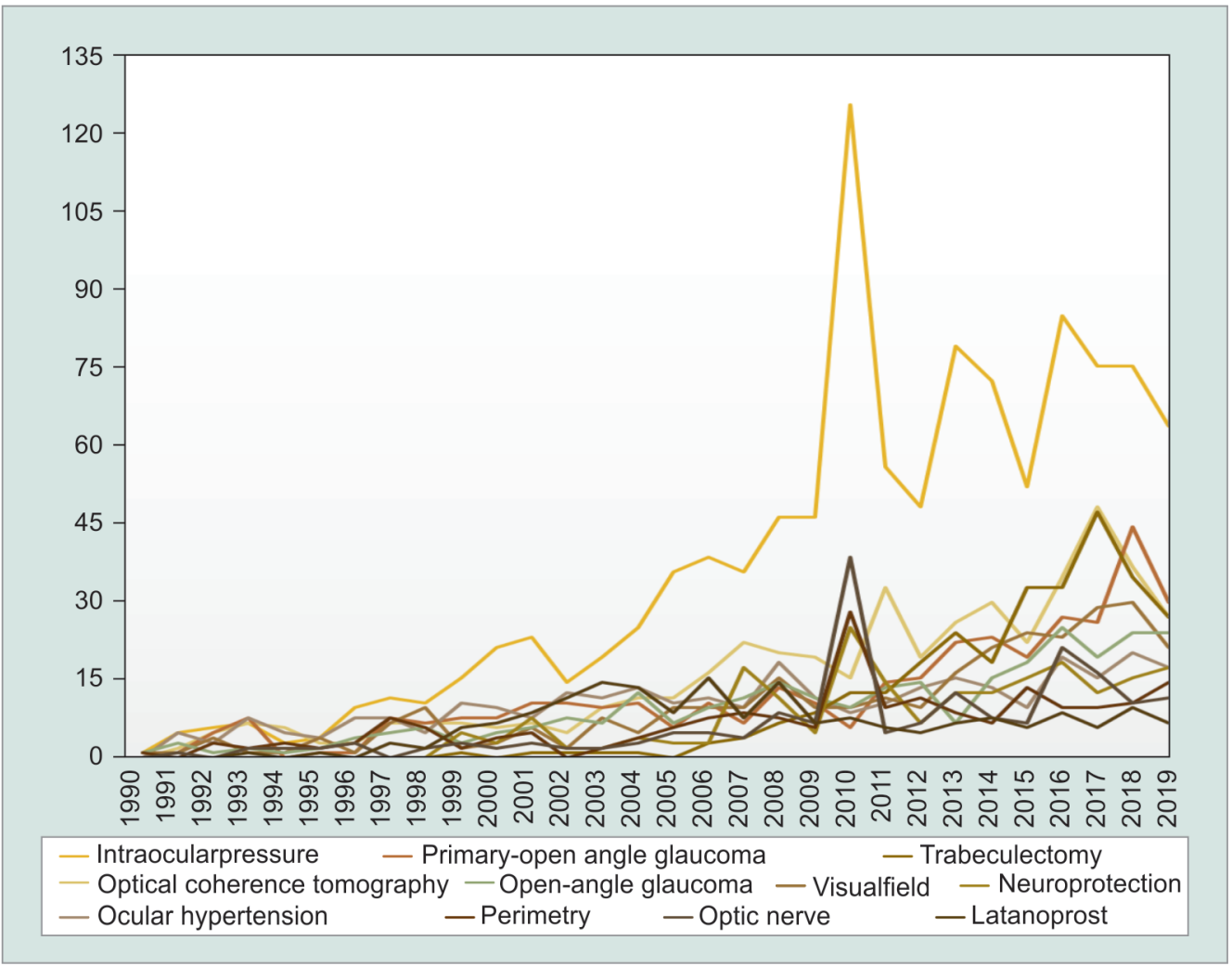

Fig. 6: Author keywords trends for top 11 keywords

Table 5: Top universities contributing glaucoma research articles

\begin{tabular}{ll}
\hline University/Institute & No. of glaucoma articles \\
\hline University of California, San Diego, USA & 934 \\
Johns Hopkins University, Baltimore, USA & 840 \\
Duke University, Durham, North Carolina, & 667 \\
USA & \\
Harvard University, Boston, USA & 577 \\
University Erlangen, Nuremberg, Bavaria, & 572 \\
Germany & \\
University of Michigan, Ann Arbor, & 534 \\
Michigan, USA & \\
L V Prasad Eye Institute, Hyderabad, India & 527 \\
Moorfields Eye Hospital, London, UK & 524 \\
University of California, Los Angeles, USA & 469 \\
All India Institute Med Sciences & 460 \\
University of lowa, lowa City, USA & 458 \\
University of Toronto, Toronto, Canada & 439 \\
University of Miami, Coral Gables, Florida, & 421 \\
USA & \\
National University of Singapore, & 399 \\
Singapore & \\
Institute of Ophthalmology & 394 \\
University of Melbourne, Melbourne, & 388 \\
Australia & 369 \\
University of Sydney, Sydney, Australia & 366 \\
Fudan University, Shanghai, China & 346 \\
Columbia University, USA & \\
Washington University, USA & \\
\hline & \\
\hline
\end{tabular}

$\mathrm{h}$-index is by far the most accepted metric to analyze the impact of scientific productions, authors, and sources. ${ }^{10,11}$ Most impactful authors in our study did not necessarily have the highest number of publications and vice versa. Notably, Harry Quigley had the highest h-index of 78 with a total of 278 publications, Robert Weinreb had the second-highest h-index of 68 with a total of 523 publications and Ritch had the third-highest h-index of 54 with 386 publications. Bengtsson appeared in our top 20 impactful authors with 87 publications while no other author on our top 20 had $<110$ publications. Also, the greatest number of author citations did not always reflect on the most author impact. For example, Spaeth and Freidman have the same $\mathrm{h}$-index while Friedman has been more frequently cited.

Although the length of the scientific career of an author influences the h-index, and may at times put newcomers at a disadvantage, additional citation parameters such as g-index, can help overcome the limitations of the h-index.

A report released by the National Eye Institute in 2012 identified certain key areas of research development in glaucoma including newer diagnostic and imaging techniques, glaucoma prevalence estimation, new IOP control therapies, ganglion cell loss as an underlying mechanism for glaucoma, genetic basis of glaucoma, novel therapeutics and anterior segment signaling pathways. ${ }^{12}$ Our study highlights similar upcoming and prevalent topics over the several years of glaucoma research while being concurrent with past contemporary studies conducted over shorter time durations. ${ }^{1}$ Expanding upon earlier findings, our study also showcases current trends in glaucoma research over the recent decade.

Another notable finding was that there were only three female authors among the top 20 authors. Although female presence has 
grown steadily in the past few decades, women still face many obstacles.

Literature databases viz., PubMed, Scopus, Google Scholar, and WoS differ in terms of their focus and coverage. One of the limitations of our study is that we used only one database, WoS, for collecting data. In our defense, we had institutional access for WoS and it offers a wider coverage and output as compared to PubMed.

\section{Implications and Future Direction}

A better understanding of the institutions and researchers focusing on glaucoma research can help researchers plan collaborations, fellowships, and research internships. Analysis of author linkages and the diversity therein must also be encouraged, across continents, to battle the global burden of disease.

It may also be prudent to analyze the reasons for the gender bias in publication and to take remedial measures to circumvent this. A step in this direction could be to encourage gender diversity in active research, as well as in leadership positions and preferential funding and grant allocations.

It is also critical to note that even though the developing world battles the socioeconomic burden of glaucoma blindness more than the developed world, research from these areas is significantly less than from the latter. It, therefore, stands to reason that glaucoma research in these areas must be fostered, both intellectually and financially, so that their issues are better understood and highlighted.

Cross-border mentorship programs and virtual classrooms may well be the way of the future, especially under the aegis of key opinion leaders as identified by the $\mathrm{h}$-index. Across the globe collaborations that share both, the technical know-how and research methodology from premium institutes, with scientists who have access to limited facilities, may well change the face of glaucoma research. This seems more feasible now than ever before, with artificial intelligence and safe electronic data transfers not being limited by geographical boundaries.

A shift to translational research reflects the changing trends, and the current focus on instrumentation in glaucoma practice is demonstrated by the disproportionate number of publications related to diagnostic equipment. While this augers well for disease diagnosis, a similar emphasis on epidemiology and quality of life concerns on management as well as the economic costs of glaucoma therapy may be the future directions for clinicianscientists across the world to focus on.

In addition, analysis of the newer metrics, such as, the download statistics, page ranks, and bookmarking tools such as Mendeley, will add interesting insight to glaucoma research and epistemology.

\section{References}

1. Ramin S, Pakravan M, Habibi G, et al. Scientometric analysis and mapping of 20 years of glaucoma research. Int J Ophthalmol 2016;9(9):1329.

2. Bradford SC. Documentation. Washington DC: Public Affairs Press; 1950. pp. 106-121.

3. Lotka AJ. The frequency distribution of scientific productivity. J Washington Acad Sci 1926;16:317-323.

4. Kumar A, Cheeseman R, Durnian JM. Subspecialization of the ophthalmic literature: a review of the publishing trends of the top general, clinical ophthalmic journals. Ophthalmology 2011;118(6):1211-1214. DOI: 10.1016/j.ophtha.2010.10.023.

5. Tham Y-C, Li X, Wong TY, et al. Global prevalence of glaucoma and projections of glaucoma burden through 2040: a systematic review and meta-analysis. Ophthalmology 2014;121(11):2081-2090. DOI: 10.1016/j.ophtha.2014.05.013.

6. Glänzel W, Thijs B. The influence of author self-citations on bibliometric macro indicators. Scientometrics 2004;59(3):281-310. DOI: 10.1023/B:SCIE.0000018535.99885.e9.

7. Glänzel W, Debackere K, Thijs B, et al. A concise review on the role of author self-citations in information science, bibliometrics and science policy. Scientometrics 2006;67(2):263-277. DOI: 10.1007/s11192-0060098-9.

8. Ogden TL, Bartley DL. The ups and downs of journal impact factors. Ann Occup Hyg 2008;52(2):73-82.

9. Ohba N, Nakao K, Isashiki Y, et al. The 100 most frequently cited articles in ophthalmology journals. Arch Ophthal 2007;125(7): 952-960. DOI: 10.1001/archopht.125.7.952.

10. Bornmann L, Daniel HD. What do we know about the $h$ index? J Am Soc Inform Sci Technol 2007;58(9):1381-1385. DOI: 10.1002/asi.20609.

11. Da Silva JAT, Dobránszki J. Multiple versions of the h-index: cautionary use for formal academic purposes. Scientometrics 2018;115(2):11071113. DOI: 10.1007/s11192-018-2680-3.

12. Vision Research: Needs, Gaps and Opportunities. Available at: https:// www.nei.nih.gov/sites/default/files/2019-06/VisionResearch2012. pdf. Accessed on 8th August, 2020. 